\title{
-Original-
}

\section{Establishment of a novel dwarf rat strain: cartilage calcification insufficient $(\mathrm{CCl})$ rats}

\author{
Masami TANAKA ${ }^{1)}$, Minoru WATANABE ${ }^{2)}$, Izuru YOKOMI ${ }^{3)}$, Naoki MATSUMOTO ${ }^{3)}$, \\ Katsuko SUDO ${ }^{4)}$, Hitoshi SATOH ${ }^{5)}$, Tsuneo IGARASHI ${ }^{6}$, Azusa SEKI ${ }^{7}$, Hitoshi AMANO $^{8)}$, \\ Kiyoshi OHURA $^{8)}$, Kakei RYU ${ }^{9}$, Shunichi SHIBATA ${ }^{10)}$, Motohiko NAGAYAMA ${ }^{11)}$, and \\ Jun-ichi TANUMA ${ }^{11)}$ \\ 1) Department of Food and Nutrition, Junior College Division, The University of Aizu, Aizu-Wakamatsu, Fukushima \\ 965-8570, Japan \\ ${ }^{2}$ Institute for Animal Experimentation, St. Marianna University Graduate School of Medicine, Kawasaki, Kanagawa \\ 216-8511, Japan \\ ${ }^{3)}$ Department of Pharmacology, St. Marianna University School of Medicine, Kawasaki, Kanagawa 216-8511, \\ Japan \\ 4) Animal Research Center, Tokyo Medical University, Tokyo 160-8402, Japan \\ ${ }^{5)}$ Laboratory of Tumor Cell Biology, Graduate School of Frontier Sciences, The University of Tokyo, Tokyo 108- \\ 8639, Japan \\ 6) Animal Care Co., Ltd., Tokyo 164-0001, Japan \\ 7) Hamri Co., Ltd. Tokyo110-0005, Japan \\ ${ }^{8)}$ Department of Pharmacology, Osaka Dental University, Osaka 573-1121, Japan \\ ${ }^{9)}$ Department of Pharmacology, Showa University School of Medicine, Tokyo 142-8555, Japan \\ 10) Maxillofacial Anatomy, Department of Maxillofacial Biology, Maxillofacial/Neck Reconstruction, Graduate \\ School, Tokyo Medical and Dental University, Tokyo 113-8549, Japan \\ 11) Department of Oral Pathology, Asahi University School of Dentistry, Hozumi, Gifu 501-0296, Japan
}

\begin{abstract}
Rats with dwarfism accompanied by skeletal abnormalities, such as shortness of the limbs, tail, and body (dwarf rats), emerged in a Jcl-derived Sprague-Dawley rat colony maintained at the Institute for Animal Experimentation, St. Marianna University Graduate School of Medicine. Since the dwarfism was assumed to be due to a genetic mutation based on its frequency, we bred the dwarf rats and investigated their characteristics in order to identify the causative factors of their phenotypes and whether they could be used as a human disease model. One male and female that produced dwarf progeny were selected, and reproduction was initiated by mating the pair. The incidence of dwarfism was $25.8 \%$ among the resultant litter, and dwarfism occurred in both genders, suggesting that it was inherited in an autosomal recessive manner. At 12 weeks of age, the body weights of the male and female dwarf rats were $40 \%$ and $57 \%$ of those of the normal rats, respectively. In soft X-ray radiographic and histological examinations, shortening and hypoplasia of the long bones, such as the tibia and femur, were observed, which were suggestive of endochondral ossification abnormalities. An immunohistochemical examination detected an aggrecan synthesis disorder, which might have led to delayed calcification and increased growth plate thickening in the dwarf rats. We hypothesized that the principal characteristics of the dwarf rats were systemically induced by insufficient cartilage calcification in their long bones; thus, we named them cartilage calcification insufficient $(\mathrm{CCl})$ rats. Key words: animal model, cartilage, calcification, dwarf, rat
\end{abstract}

(Received 25 August 2014 / Accepted 15 October 2014 / Published online in J-STAGE 20 December 2014)

Address corresponding: M. Tanaka, Department of Food and Nutrition, Junior College Division, The University of Aizu, Aizu-Wakamatsu, Fukushima 965-8570, Japan

(C)2015 Japanese Association for Laboratory Animal Science 


\section{Introduction}

Bone development occurs via two processes, intramembranous and endochondral ossification. Most skeletal elements, including vertebrae and long bones, are formed via endochondral ossification. Cartilage tissue is found at either end of long bones in regions called growth plates. Long bones grow when the chondrocytes in their growth plates divide and increase in number. The cartilage tissue in growth plates is composed of specialized chondroprogenitor cells called chondroblasts, which produce a large amount of extracellular matrix molecules including type II collagen fibers, an abundant proteoglycan-rich ground substance, and elastin fibers.

In humans, the gene mutation-associated constant activation of fibroblast growth factor receptor 3 (FGFR3) is known to be a causative factor in achondroplasia, cartilage hypoplasia, and lethal osteodysplasty, all of which can lead to micromelic dwarfism $[12,15]$. Experimental FGFR3 activation in animals induced micromelic dwarfism, and abnormal differentiation, growth, and ossification of growth plate cartilage were noted [22]. C-type natriuretic peptide (CNP) inhibits FGFR3 signaling-induced activation of the mitogen-activated protein kinase (MAPK) pathway [27], and CNP-knockout animals develop achondroplasia-like dwarfism [5]. KMI rats lack cyclic GMP-dependent protein kinase II (cGKII) activity, which is presumed to be a downstream signal of CNP, due to a point mutation in the $c$ GKII gene and are used as a spontaneous micromelic dwarfism model [4]. Many factors and mechanisms are associated with morphological changes in the differentiation, growth, and ossification of growth plate cartilage and have been investigated by preparing animal models in which these factors have been genetically modified [7]. On the other hand, there are several animal models of spontaneous micromelic dwarfism [3, 5, 19-21]. The preparation of a novel animal model of spontaneous micromelic dwarfism and the elucidation of the cause of such dwarfism would contribute to determining the pathology of micromelic dwarfism and the mechanisms responsible for the differentiation, growth, and ossification of growth plate cartilage. Rats with dwarfism accompanied by skeletal abnormalities, such as shortness of the limbs, tail, and body (dwarf rats), emerged in a Jcl-derived Sprague-Dawley rat colony maintained at the Institute for Animal Experimentation, St. Marianna University Graduate School of Medicine. In this study, since we hypothesized that the principal characteristic of the dwarf rats was an aggrecan synthesis disorder, which might have led to delayed calcification and increased growth plate thickening in their long bones, we named them cartilage calcification insufficient (CCI) rats.

\section{Materials and Methods}

\section{Rats}

The proband rats were a male and female derived from a colony of Jcl-derived Sprague-Dawley (SD) rats. They had been maintained at our university and had produced dwarf progeny. The animal room was controlled at a temperature of $22 \pm 2{ }^{\circ} \mathrm{C}$ and $55 \pm 5 \%$ humidity with 12 $\mathrm{h}$ of lighting (lights on at 6:00 am). The animals were given free access to food and water. Plastic cages (W270 $\times$ L440 $\times$ H1 $87 \mathrm{~mm}$ ) and wooden bedding chips (White Flake; Oriental Yeast, Tokyo, Japan) were used for maintenance.

All study protocols involving the use of animals were reviewed and approved by the institutional animal research committee and the president of St. Marianna University School of Medicine.

\section{Breeding}

A male and female that had produced dwarf progeny were selected, and breeding was initiated by mating them. Their progeny were weaned at 4 weeks after birth, which is when the dwarf rats were able to grow independently. Wild type rats and heterozygote rats $(+/ c c i)$ were morphologically normal (normal rats) and selected using the progeny test, and one-to-one mating of the heterozygote $(+/ \mathrm{cci})$ males and females was initiated at 12 weeks after birth.

\section{Phenotypic characterization}

All rats were weighed every week and had their total length (from nose to tail tip), body length (from nose to anus), tail length, and head length measured at 4 weeks after birth.

\section{Soft $X$-ray analysis}

Carcasses of 4-week-old rats were fixed in $4 \%$ neutral buffered glutaraldehyde solution after the skin and visceral organs had been removed [2]. The head and the bilateral femurs and tibias were excised. Radiograms were acquired using a soft X-ray apparatus (SOFTEX- 
CMB; SOFTEX, Tokyo, Japan; $40 \mathrm{KV}, 3.0$ mAs, irradiation distance of $90 \mathrm{~cm}$, exposure period of $40 \mathrm{sec}-$ onds).

\section{Tissue preparation}

Specimens were embedded in Epon 812 (TAAB, Berkshire, UK ) without decalcification. Two-micrometerthick sections were stained with toluidine blue and von Kossa stain, as described previously [16]. Other specimens were fixed with $4 \%$ paraformaldehyde $(0.1 \mathrm{M}$ phosphate buffer, $\mathrm{pH} 7.4$, room temperature), decalcified in $10 \%$ ethylenediaminetetraacetic acid for 21 days at room temperature, and embedded in paraffin using standard procedures. Serial $5-\mu \mathrm{m}$-thick sections were used for immunohistochemistry.

\section{Immunohistochemistry}

A monoclonal antibody against aggrecan (12/21/1C6) was purchased from the Developmental Studies Hybridoma Bank (Iowa City, IA, USA). This antibody was utilized in our previous study [2]. Before the primary antibody reaction, all of the sections were digested with $25 \mathrm{mg} / \mathrm{ml}$ testicular hyaluronidase (Sigma Chemical Co., St. Louis, MO, USA) in phosphate buffered saline for $30 \mathrm{~min}$ at $37^{\circ} \mathrm{C}$; and then were reduced and alkylated as described previously [17]. The streptavidin-biotin method was then performed using a Histofine SAB kit (Nichirei, Tokyo, Japan) as described previously [2]. The sections were treated with 3-amino-9-ethylcarbazole to develop the chromogen reaction. The negative control sections were incubated with normal mouse IgG instead of the primary antibody. The sections were examined after being counterstained with hematoxylin.

\section{Statistical analysis}

Data are expressed as mean $\pm \mathrm{SD}$ values. The statistical significance of differences was evaluated by analysis of variance (ANOVA) followed by Bonferroni's test and the Chi-square test. Differences were considered to be significant at $P<0.05$.

\section{Results}

\section{Hereditary}

The overall incidence of dwarfism in the litters produced in this study was $25.8 \%$ (male, $26.3 \%$; female, $25.4 \%$, and dwarfism developed in both genders. This suggested that the dwarfism was inherited in an
Table 1. Analysis of the modes of inheritance in the CCI rat

\begin{tabular}{ccccc}
\hline \multirow{2}{*}{$\begin{array}{c}\text { Total number } \\
\text { of deliveries }\end{array}$} & \multicolumn{3}{c}{ Number of pups } & Segregation ratio \\
\cline { 2 - 4 } & Normal & Dwarf & Total & Normal/Dwarf \\
\hline \multirow{2}{*}{ Male } & $\begin{array}{l}628 \\
+/+\end{array}$ & $\begin{array}{c}224 \\
\text { cci/cci }\end{array}$ & 852 & 2.80 \\
& $+/ c c i$ & & & \\
\hline \multirow{2}{*}{ Female } & $\begin{array}{l}622 \\
+/+\end{array}$ & 212 & 834 & 2.93 \\
& $+/ c c i$ & & & \\
\hline 200 & 1,250 & 436 & 1,686 & 2.87 \\
\hline
\end{tabular}

Spontaneously mutant CCI rats that showed dwarfism accompanied by skeletal abnormality such as shortness of the four limbs. Heterozygote rats $(+/ c c i)$ were selected by the progeny test, and one-to-one mating of the heterozygote males and females was performed for the analysis of the modes of inheritance. The value of the segregation ratio (2.87) shows that the modes of inheritance of the CCI phenotype (cci expression) is an autosomal recessive inheritance. There was no difference in the incidence of dwarfism between male and female $\mathrm{CCI}$ rats (Chi-square test, $P=0.689$ ).

autosomal recessive manner (Table 1). There was no difference in the incidence of dwarfism between male and female CCI rats (Chi-square test, $P=0.689$ ).

No progeny were obtained by mating male CCI rats with female CCI rats or male or female CCI rats with normal rats, confirming that the CCI rats were infertile or unable to reproduce.

\section{Growth and body weight}

The body weight variations observed from 3 to 12 weeks after birth are shown in Fig. 1. The body weight of the CCI rats was markedly lower than that of the normal rats at 3 weeks after birth, and the difference increased with age. At 12 weeks after birth, the body weights of the male and female CCI rats were $40 \%$ and $57 \%$ of those of the normal rats, respectively.

\section{Phenotypic characterization}

A macroscopic examination and morphometric analysis of the CCI rats detected dwarfism accompanied by skeletal abnormalities, such as shortness of the limbs (all four limbs), tail, and body (Figs. 2A-C). In a comparison between the normal and CCI rats based on soft X-ray radiography performed at 4 weeks after birth, it was demonstrated that the limbs and vertebrae of the CCI rats were markedly shorter than those of the normal rats (Figs. 3A-C). The CCI rats also had shorter heads than the normal rats at 4 weeks after birth (Figs. 3D and E). 


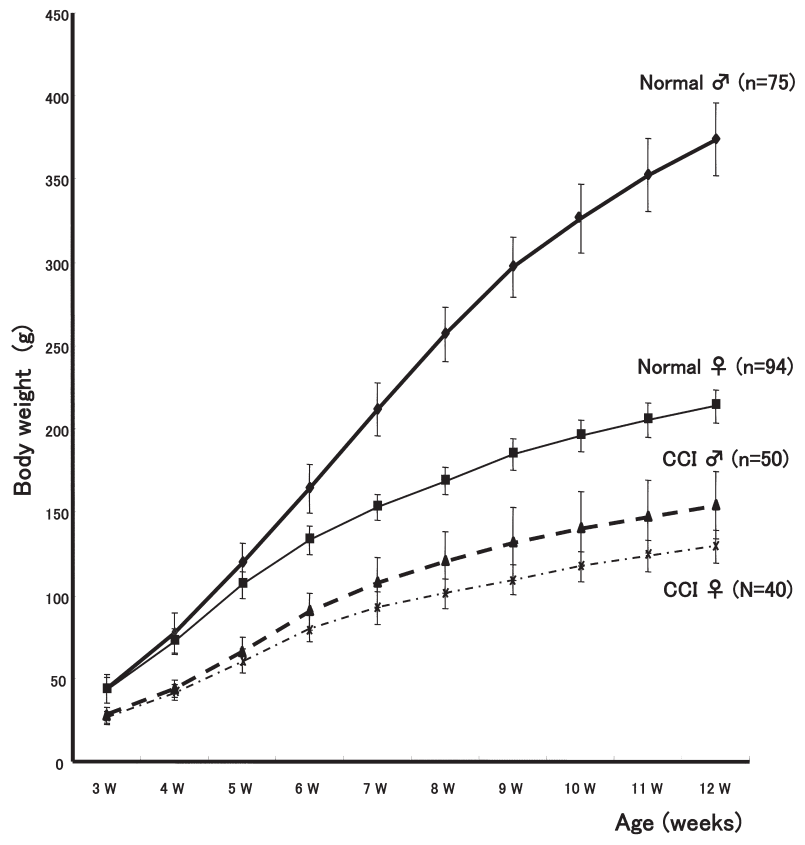

Fig. 1. Growth curves showing the body weights of the normal and CCI rats.
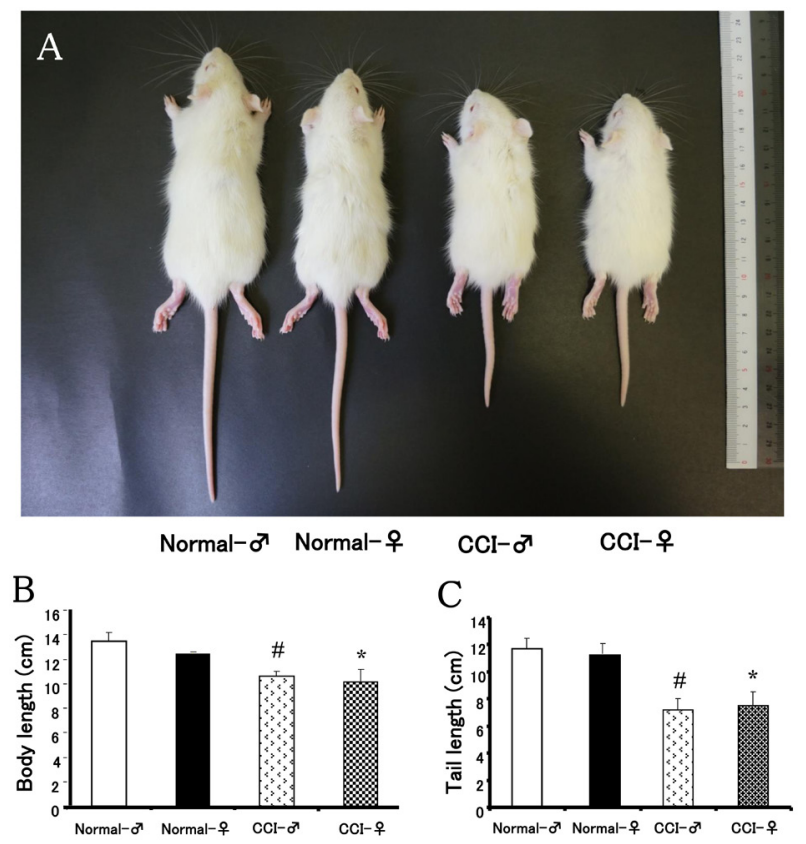

Fig. 2. Macroscopic findings regarding body length (A, B) and tail length $(\mathrm{A}, \mathrm{C})$. Data are expressed as mean $\pm \mathrm{SD}$ values of normal male $(\mathrm{n}=12)$, normal female $(\mathrm{n}=9)$, CCI male $(\mathrm{n}=6)$, and CCI female $(\mathrm{n}=6)$ rats. ${ }^{\#} P<0.01$ vs. normal male rats. ${ }^{*} P<0.01$ vs. normal female rats.

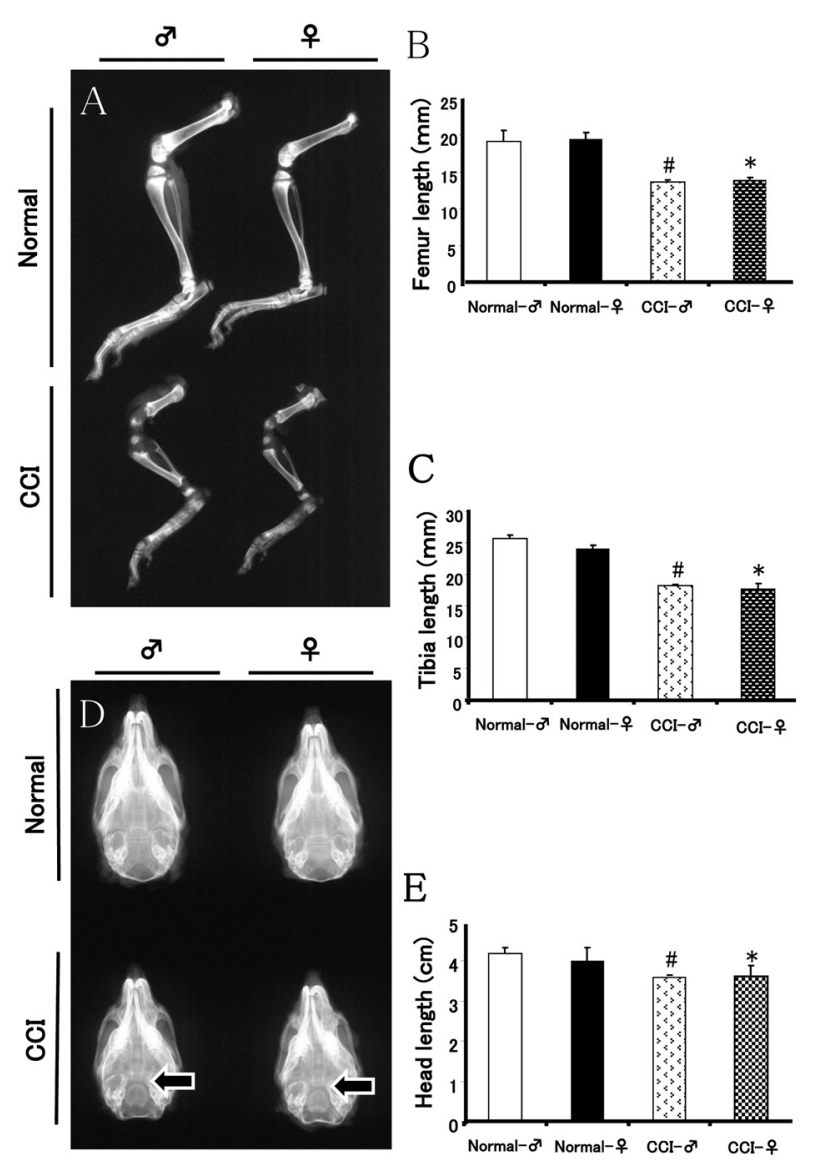

Fig. 3. Radiographic findings regarding the lengths of the femur $(\mathrm{A}, \mathrm{B})$, tibia $(\mathrm{A}, \mathrm{C})$, and head $(\mathrm{D}, \mathrm{E})$. Both male and female $\mathrm{CCI}$ rats exhibited delayed posterior fontanel closing (arrows in D). Data are expressed as mean $\pm \mathrm{SD}$ values of normal male $(n=12)$, normal female $(n=9)$, CCI male $(n=6)$, and CCI female $(\mathrm{n}=6)$ rats. ${ }^{\#} P<0.01$ vs. normal male rats. $* P<0.01$ vs. normal female rats.

Moreover, it became clear that both the male and female CCI rats exhibited delayed anterior fontanel closing (Fig. 3D). On the other hand, the long bones of the CCI rats had markedly greater radiolucent spaces among their growth plate cartilage than those of the normal rats, which was indicative of insufficient cartilage calcification (Fig. 3A).

\section{Von Kossa staining findings}

Examinations of sections that had been stained with von Kossa stain indicated that the calcified matrix was located in the lowest part of the growth plate in the normal rats and that it was contiguous with the bone spicules in the primary spongiosa (Fig. 4A), which were arranged in an orderly manner along the long axis of the tibia 

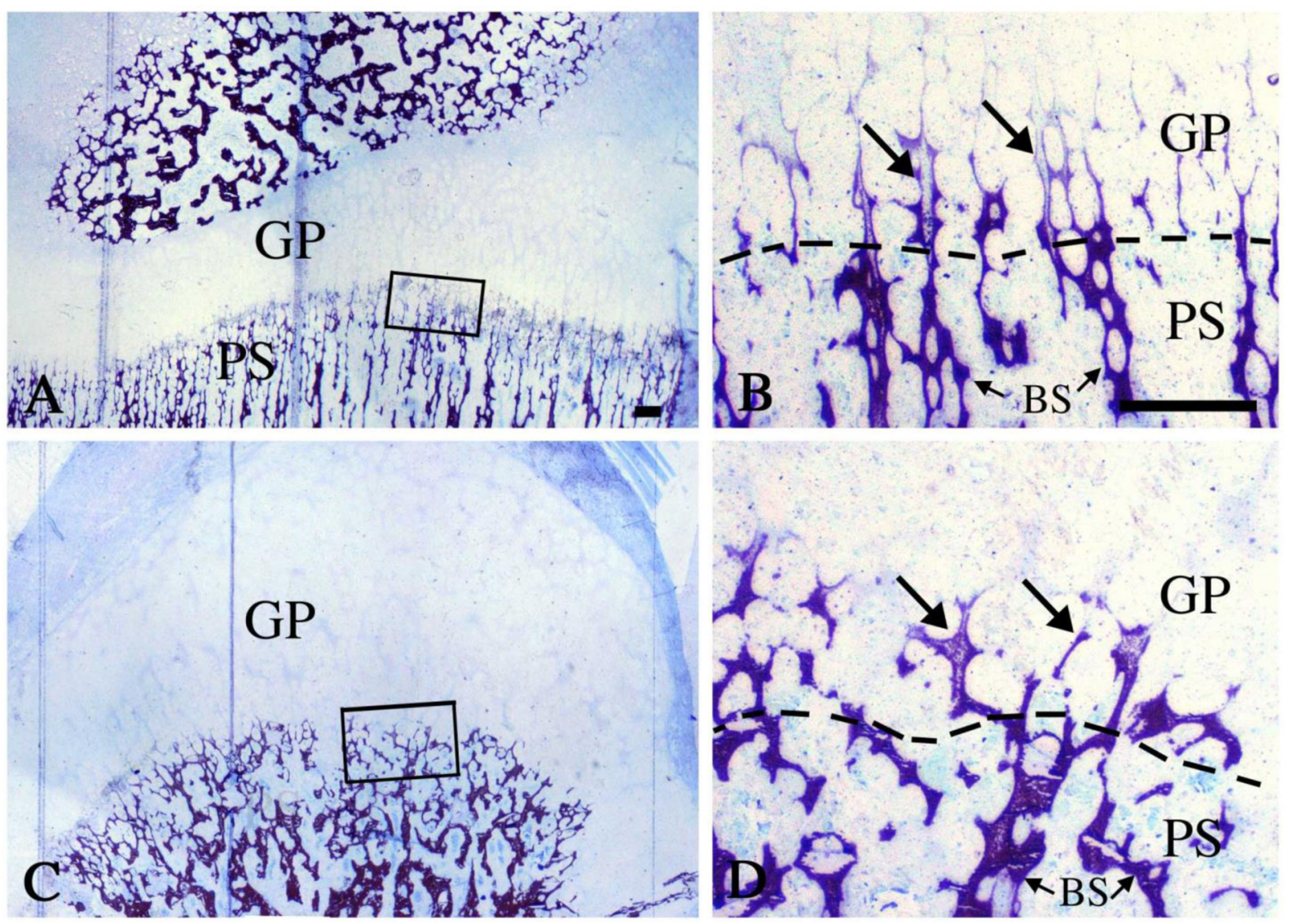

Fig. 4. Undecalcified sections of the tibial knee joints of a normal rat (A, B) and a CCI rat (C, D) that had been stained with toluidine blue and von Kossa stain. B and D show magnified views of the rectangular areas shown in A and C, respectively. The dotted lines indicate the erosion zone, i.e., the border between the growth plate (GP) and the primary spongiosa (PS). (A, B) A calcified matrix (arrows) was detected in the lowest part of the growth plate (GP), which was contiguous with the bone spicules (BS) in the primary spongiosa (PS). The bone spicules were arranged in an orderly manner along the long axis of the tibia. (C, D) The bone spicules (BS) were arranged randomly and were thicker that those seen in the normal rats. Staining intensity did not differ between the normal and CCI rats (compared with the BS in B). We obtained the same results when we examined three different samples $(n=3)$. Bar $=200 \mu \mathrm{m}$

(Fig. 4B). Conversely, the bone spicules of the CCI rats were arranged randomly and were thicker than those observed in the normal rats. However, the staining intensity did not differ from that seen in the normal rats (Figs. 4C and D).

\section{Histological and immunohistochemical findings}

Examinations of tibial knee joints demonstrated that the articular cartilage and growth plate were clearly distinguishable and that the growth plate cartilage had been organized into a well-established zone at 4 weeks after birth in the normal rats (Fig. 5A). However, the secondary ossification center had not fully formed, and thicker growth plates were observed in the CCI rats. The growth plate cartilage was also arranged in a disordered manner in the CCI rats (Fig. 5B). Aggrecan immunostaining was detected in both the articular cartilage and growth plate in the normal rats (Figs. 5C and D). At higher magnification, it was found that the aggrecan immunostaining was evenly distributed throughout the growth plate (arrow in Fig. 5D). In the CCI rats, aggrecan was unevenly distributed throughout the cartilage tissue (Figs. 5E and F). In particular, an aggrecan-negative area was observed in the middle of the growth plate (arrow in Fig. 5F).

\section{Discussion}

Many spontaneously mutant rat strains [5, 20, 22, 27] have been established as human disease models and used for etiological studies and to facilitate the development 


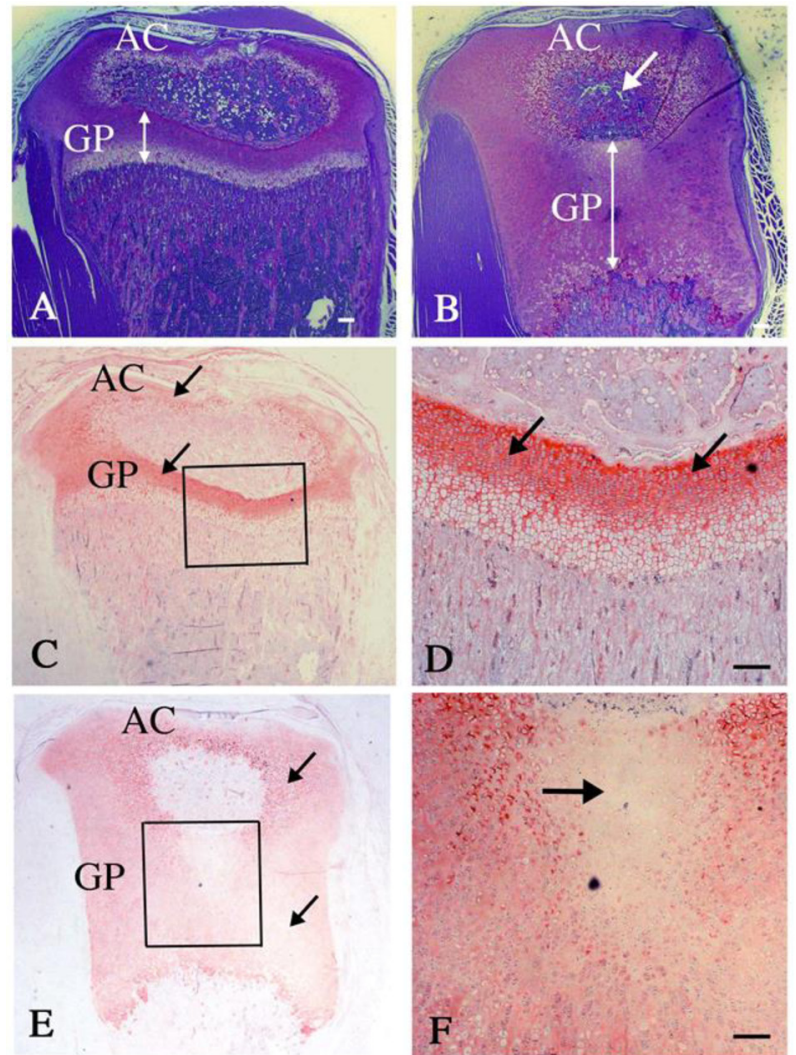

Fig. 5. Longitudinal sections from the tibial growth plates of normal rats $(\mathrm{A}, \mathrm{C}, \mathrm{D})$ and $\mathrm{CCI}$ rats $(\mathrm{B}, \mathrm{E}, \mathrm{F})$ that had been stained with toluidine blue (A, B) or immunostaining for aggrecan $(\mathrm{C}-\mathrm{F})$. D and $\mathrm{F}$ show magnified views of the rectangular areas shown in $\mathrm{C}$ and $\mathrm{E}$, respectively. The articular cartilage ( $\mathrm{AC}$ in $\mathrm{A}$ ) and growth plate ( $\mathrm{GP}$ in $\mathrm{A})$ in normal rats were clearly distinguishable, and the architecture of the chondrocyte zones in the growth plate cartilage (two-way arrow) was well established in the normal rats. The secondary ossification center in CCI rats (arrow in B) had not fully formed, and the growth plate (GP, two-way arrow in $\mathrm{B}$ ) in the CCI rats was longer than that in normal rats (GP in $\mathrm{A})$. The architecture of the chondrocyte zones was irregular or disorganized in the CCI rats. In the normal rats, aggrecan immunostaining (arrows in $\mathrm{C}$ ) was detected in the articular cartilage ( $\mathrm{AC}$ in $\mathrm{C}$ ) and the growth plate (GP in C). At higher magnification, aggrecan immunostaining was evenly distributed throughout the growth plate (arrows in D). In the CCI rats, aggrecan immunostaining (arrows in E) was unevenly distributed throughout the articular cartilage (AC in E) and growth plate (GP in E). At higher magnification, an aggrecan-negative area was also observed (arrow in F). We obtained the same results when we examined three different samples $(\mathrm{n}=3)$. Bars $=100 \mu \mathrm{m}$

of new medicines and treatments. Since the dwarfism observed in the CCI rats used in the present study was assumed to be due to a genetic mutation based on its frequency, we bred the rats with this dwarfism and in- vestigated their characteristics to identify the causative factors of their condition and whether they could be used as a human disease model. Endochondral ossification was markedly delayed in the dwarf rats, which makes these rats different from other animal models of dwarfism in the manner of the cartilage and bone maturation [5, $20,22,27]$. The CCI rats did not typically show histological findings such as cracks or fissures at the articular surface, which was also quite different from osteoarthritic model mouse strains [26].

Different from the normal rats, the CCI rats did not exhibit any gender differences in body, tail, or bone length during the early stages of their growth. However, the homozygous mutants of the Komeda Miniature Rat Ishikawa (KMI rat) strain exhibited growth equivalent to 70 to $80 \%$ of that of both sexes of heterozygous animals and retained the gender differences during growth $[4,20]$. The cartilage matrix deficiency $(\mathrm{cmd})$ mouse is a natural aggrecan gene knockout mouse, and homozygotes $(\mathrm{cmd} / \mathrm{cmd})$ show severe dwarfism and immediately die after birth. Even heterozygous mice $(+/ \mathrm{cm} d)$ show slight dwarfism, resembling CCI rats in the present study $[24,25]$. Thus an aggrecan synthesis disorder, which might lead to delayed endochondral ossification and increased growth plate thickening, might be a reason for dwarfism in the CCI rats.

The SRY gene provides instructions for making a transcription factor called the sex-determining region $\mathrm{Y}$ protein. It contains a high-mobility group (HMG)-box DNA-binding domain characteristic of the SOX family of transcription factors. The SRY protein causes a fetus to develop as a male. The related autosomal gene $\operatorname{Sox} 9$ is also known from loss-of-function mutations in mice and humans to be essential for Sertoli cell [14] and chondrocyte differentiation [1]. Thus, the gene carrying the mutation responsible for the CCI phenotype might be involved in determining gender differences in endochondral ossification and skeletal growth.

As a result of mating, both male and female CCI rats were infertile (data not shown). The causes of infertility of the CCI rats is being investigated. The vaginal smears of female CCI rats demonstrated an abnormal sexual cycle. Other dwarf rat strains, the growth hormone-deficient, spontaneous dwarf rat (SDR) [3] and prkg genemutated KMI rat [11], were fertile. Thus, infertility was the major characteristic of the CCI rats. The reason for infertility in CCI rats is unknown. Several kinds of glycoproteins in the zona pellucida (ZP) around the egg, 
however, are essential for binding of sperm with the ZP $[18,23]$. Thus, this aggrecan, impaired synthesis of glycoproteins may be associated with infertility of CCI rats. The body weight of CCI rats was significantly lower than that of the normal control rats (at eight weeks of age, $46 \%$ (males) and 53\% (females) of the normal rats). KMI rats presented similar abnormalities in the growth plate cartilage and less pronounced dwarfism compared with the CCI rats (at eight weeks of age, $70-80 \%$ of the body weight of the normal rats). The CCI rats were also characterized by marked dwarfism.

An aggrecan synthesis disorder, which might have led to delayed calcification and increased the growth plate and articular cartilage thickening, was detected in the CCI rats by the immunohistochemical examination. Thus, a deficiency of aggrecan affects chondrocyte maturation in the growth plate and articular cartilage, leading to the arrest of long bone maturation $[9,13]$. The head length of the CCI rats was also affected by a delay in age-dependent cartilage growth and ossification at the cranial base synchondrosis, which is composed of sphenoethmoidal, intrasphenoidal, and spheno-occipital synchondroses [10]. Chondrocyte disorganization due to molecular disorders such as aberrant hedgehog and Wnt canonical signaling in cartilage tissue might be partially responsible for the phenotypes of CCI rats $[6$, 8]. Phenotypic variations might also be induced by differences in genetic background, although advanced genomic analysis is required to confirm this. Since we hypothesized that the principal characteristics of the dwarf rats was an aggrecan synthesis disorder, which might have led to delayed calcification and increased growth plate thickening in their long bones, we named them cartilage calcification insufficient (CCI) rats.

\section{Acknowledgments}

This study was supported by JSPS KAKENHI Grant Number 20591792.

\section{References}

1. Akiyama, H., Chaboissier, M.C., Martin, J.F., Schedl, A., and de Crombrugghe, B. 2002. The transcription factor Sox9 has essential roles in successive steps of the chondrocyte differentiation pathway and is required for expression of Sox 5 and Sox6. Genes Dev. 16: 2813-2828. [Medline] [CrossRef]

2. Amano, H. 1989. A histomorphometric analysis of the alveolar bone resorption process in calcium-deficient rats. Shika
Kiso Igakkai Zasshi 31: 404-416. [Medline]

3. Charlton, H.M., Clark, R.G., Robinson, I.C., Goff, A.E., Cox, B.S., Bugnon, C., and Bloch, B.A. 1988. Growth hormonedeficient dwarfism in the rat: a new mutation. J. Endocrinol. 119: 51-58. [Medline] [CrossRef]

4. Chikuda, H., Kugimiya, F., Hoshi, K., Ikeda, T., Ogasawara, T., Shimoaka, T., Kawano, H., Kamekura, S., Tsuchida, A., Yokoi, N., Nakamura, K., Komeda, K., Chung, U.I., and Kawaguchi, H. 2004. Cyclic GMP-dependent protein kinase II is a molecular switch from proliferation to hypertrophic differentiation of chondrocytes. Genes Dev. 18: 2418-2429. [Medline] [CrossRef]

5. Chusho, H., Tamura, N., Ogawa, Y., Yasoda, A., Suda, M., Miyazawa, T., Nakamura, K., Nakao, K., Kurihara, T., Komatsu, Y., Itoh, H., Tanaka, K., Saito, Y., Katsuki, M., and Nakao, K. 2001. Dwarfism and early death in mice lacking C-type natriuretic peptide. Proc. Natl. Acad. Sci. USA 98: 4016-4021. [Medline] [CrossRef]

6. Koyama, E., Young, B., Nagayama, M., Shibukawa, Y., Enomoto-Iwamoto, M., Iwamoto, M., Maeda, Y., Lanske, B., Song, B., Serra, R., and Pacifici, M. 2007. Conditional Kif3a ablation causes abnormal hedgehog signaling topography, growth plate dysfunction, and excessive bone and cartilage formation during mouse skeletogenesis. Development 134 : 2159-2169. [Medline] [CrossRef]

7. Mackie, E.J., Tatarczuch, L., and Mirams, M. 2011. The skeleton: a multi-functional complex organ: the growth plate chondrocyte and endochondral ossification. J. Endocrinol. 211: 109-121. [Medline] [CrossRef]

8. Michigami, T. 2013. Regulatory mechanisms for the development of growth plate cartilage. Cell. Mol. Life Sci. 70: 4213-4221. [Medline] [CrossRef]

9. Domowicz, M.S., Cortes, M., Henry, J.G., and Schwartz, N.B. 2009. Aggrecan modulation of growth plate morphogenesis. Dev. Biol. 329: 242-257. [Medline] [CrossRef]

10. Nagayama, M., Iwamoto, M., Hargett, A., Kamiya, N., Tamamura, Y., Young, B., Morrison, T., Takeuchi, H., Pacifici, M., Enomoto-Iwamoto, M., and Koyama, E. 2008. Wnt/ $\beta$-catenin signaling regulates cranial base development and growth. J. Dent. Res. 87: 244-249. [Medline] [CrossRef]

11. Nogami, H., Watanabe, T., and Takeuchi, T. 1992. Effect of growth hormone $(\mathrm{GH})$ on the promotion of body weight gain in the spontaneous dwarf rat: a novel experimental model for isolated GH deficiency. Horm. Metab. Res. 24: 300-301. [Medline] [CrossRef]

12. Rousseau, F., Bonaventure, J., Legeai-Mallet, L., Pelet, A., Rozet, J.M., Maroteaux, P., Le Merrer, M., and Munnich, A. 1996. Mutations of the fibroblast growth factor receptor-3 gene in achondroplasia. Horm. Res. 45: 108-110. [Medline] [CrossRef]

13. Sato, T., Kudo, T., Ikehara, Y., Ogawa, H., Hirano, T., Kiyohara, K., Hagiwara, K., Togayachi, A., Ema, M., Takahashi, S., Kimata, K., Watanabe, H., and Narimatsu, H. 2011. Chondroitin sulfate $N$-acetylgalactosaminyltransferase 1 is necessary for normal endochondral ossification and aggrecan metabolism. J. Biol. Chem. 286: 5803-5812. [Medline] [CrossRef]

14. Sekido, R. and Lovell-Badge, R. 2008. Sex determination in- 
volves synergistic action of SRY and SF1 on a specific Sox9 enhancer. Nature 453: 930-934. [Medline] [CrossRef]

15. Shiang, R., Thompson, L.M., Zhu, Y.Z., Church, D.M., Fielder, T.J., Bocian, M., Winokur, S.T., and Wasmuth, J.J. 1994. Mutations in the transmembrane domain of FGFR3 cause the most common genetic form of dwarfism, achondroplasia. Cell 78: 335-342. [Medline] [CrossRef]

16. Shibata, S., Baba, O., Ohsako, M., Shikano, S., Terashima, T., Yamashita, Y., and Ichijo, T. 1991. Histological observation of large light cells that seem to be surviving hypertrophic chondrocytes in the rat mandibular condyle. Arch. Oral Biol. 36: 541-544. [Medline] [CrossRef]

17. Shibata, S., Suda, N., Fukada, K., Ohyama, K., Yamashita, Y., and Hammond, V.E. 2003. Mandibular coronoid process in parathyroid hormone-related protein-deficient mice shows ectopic cartilage formation accompanied by abnormal bone modeling. Anat. Embryol. (Berl.) 207: 35-44. [Medline] [CrossRef]

18. Shur, B.D., Evans, S., and Lu, Q. 1998. Cell surface galactosyltransferase: current issues. Glycoconj. J. 15: 537-548. [Medline] [CrossRef]

19. Takeuchi, T., Suzuki, H., Sakurai, S., Nogami, H., Okuma, S., and Ishikawa, H. 1990. Molecular mechanism of growth hormone $(\mathrm{GH})$ deficiency in the spontaneous dwarf rat: detection of abnormal splicing of GH messenger ribonucleic acid by the polymerase chain reaction. Endocrinology 126 : 31-38. [Medline] [CrossRef]

20. Tsuchida, A., Yokoi, N., Namae, M., Fuse, M., Masuyama, T., Sasaki, M., Kawazu, S., and Komeda, K. 2008. Phenotypic characterization of the Komeda miniature rat Ishikawa, an animal model of dwarfism caused by a mutation in Prkg2. Comp. Med. 58: 560-567. [Medline]

21. Tsuji, T. and Kunieda, T. 2005. A loss-of-function mutation in natriuretic peptide receptor $2(\mathrm{Npr} 2)$ gene is responsible for disproportionate dwarfism in $\mathrm{cn} / \mathrm{cn}$ mouse. J. Biol. Chem. 280: 14288-14292. [Medline] [CrossRef]

22. Wang, Y., Spatz, M.K., Kannan, K., Hayk, H., Avivi, A., Gorivodsky, M., Pines, M., Yayon, A., Lonai, P., and Givol, D. 1999. A mouse model for achondroplasia produced by targeting fibroblast growth factor receptor 3. Proc. Natl. Acad. Sci. USA 96: 4455-4460. [Medline] [CrossRef]

23. Wassarman, P.M. 1990. Profile of a mammalian sperm receptor. Development 108: 1-17. [Medline]

24. Watanabe, H., Nakata, K., Kimata, K., Nakanishi, I., and Yamada, Y. 1997. Dwarfism and age-associated spinal degeneration of heterozygote cmd mice defective in aggrecan. Proc. Natl. Acad. Sci. USA 94: 6943-6947. [Medline] [CrossRef]

25. Watanabe, H. and Yamada, Y. 2002. Chondrodysplasia of gene knockout mice for aggrecan and link protein. Glycoconj. J. 19: 269-273. [Medline] [CrossRef]

26. Yamamoto, H. and Iwase, N. 1998. Spontaneous osteoarthritic lesions in a new mutant strain of the mouse. Exp. Anim. 47: 131-135. [Medline] [CrossRef]

27. Yasoda, A., Komatsu, Y., Chusho, H., Miyazawa, T., Ozasa, A., Miura, M., Kurihara, T., Rogi, T., Tanaka, S., Suda, M., Tamura, N., Ogawa, Y., and Nakao, K. 2004. Overexpression of CNP in chondrocytes rescues achondroplasia through a MAPK-dependent pathway. Nat. Med. 10: 80-86. [Medline] [CrossRef] 